\title{
Sociología médica: origen y campo de acción
}

\section{Medical sociology: origin and field of action}

\author{
Gunther Balarezo-López \\ Recibido 20 mayo 2014 / Enviado para modificación 23 junio 2015 / Aceptado 12 octubre 2017
}

\section{RESUMEN}

Se hizo una revisión de la literatura publicada sobre el origen de la sociología médica y su campo de acción. Para ello, se analizaron diversas publicaciones y se resumieron los aspectos más relevantes. A pesar de que la enfermedad siempre ha estado ligada a aspectos socioculturales, recién a mediados del siglo XX, la medicina reconoce la importancia de la sociología en la explicación de temas relacionados a la salud, especialmente para explicar los determinantes sociales de la salud. En la actualidad, los sociólogos trabajan de manera multidisciplinaria con médicos para investigar y analizar cuestiones concernientes a la salud de las personas, para mejorar el bienestar y la calidad de vida de la población. En este sentido, el aporte de la sociología no solo ha enriquecido la comprensión de algunas enfermedades, sino también diferentes aspectos en el quehacer de la medicina.

Palabras Clave: Sociología médica; determinantes sociales de la salud (fuente: DeCS, BIREME).

\section{ABSTRACT}

A review of the published literature on the origin of medical sociology and its field of action was made. For this, several publications were analyzed and the most relevant aspects were summarized. Although the concept of disease has always been linked to sociocultural aspects, only until the mid-twentieth century, Medicine recognized the importance of sociology to explain health-related issues, especially the social determinants of health. Currently, sociologists work in a multidisciplinary way with doctors to investigate and analyze issues concerning the health of people, to improve the welfare and quality of life of the population. In this sense, the contribution of Sociology has not only enriched the understanding of some diseases, but also different aspects of the work of Medicine.

Key Words: Medical sociology; social determinants of health (source: MeSH, NLM).

$\mathrm{L}$ a naturaleza de la sociedad fue uno de los más importantes temas de reflexión para los grandes pensadores de la antigüedad. Así se tiene que el filósofo chino Confucio (55 1-479 a.C.), los filósofos griegos Platón (427-347 a.C.) y Aristóteles (384-322 a.C.), el filósofo y teólogo medieval Santo Tomás de Aquino (1225-1274), el filósofo árabe Ibn Jaldún (1332-1406) y el político francés Montesquieu (1689-1755), dedicaron gran parte de sus reflexiones al tema de la sociedad ( 1 ).

Posteriormente, a mediados del siglo x IX surge una disciplina científica que estudia la sociedad. Aparece como consecuencia de la gran crisis de carácter internacional que fue la Revolución Francesa de 1789 y de la fuerte influencia del pensamiento "socialista" de Saint Simón (uno de los tres clásicos representantes del "socialismo utópico" con Fourier y Owen) así como de la capacidad creadora de Augusto Comte para interpretar el anhelo colectivo que pugnaba por el advenimiento de un nuevo estado social (2).

En tal sentido, el término sociología, fue acuñado por primera vez por el ensayista francés Emmanuel Joseph Sieyès (1748-1836), del latín Socius (compañero) y el sufi-
GB: Sociólogo. M. Sc. Salud Pública, Docente. Escuela de Medicina, Universidad Peruana de Ciencias Aplicadas. Lima, Perú. gunther.balarezo@upc.edu.pe 


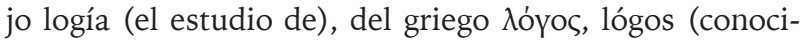
miento). El término fue independientemente reinventado e introducido por Augusto Comte en su Filosofía Positiva (1842), quien al inicio había tratado de adjudicarle el calificativo de Física Social, pero por aquella época el sabio belga Adolfo Quetelet, había empezado a publicar bajo este rubro algunos trabajos de investigación aplicando la estadística a los hechos sociales. Por este motivo, Comte en la búsqueda de un nuevo nombre le puso el de Sociología. Esta palabra es un neologismo, porque es una combinación del latín y griego y sus dos componentes expresan el objetivo de la nueva ciencia: Socios, que hace referencia a la sociedad y logia que significa estudio en un nivel elevado; por lo que etimológicamente sociología es el estudio de la sociedad en un nivel muy alto de generalización o abstracción $(2,3)$.

Por otro lado, se menciona que la palabra Sociología fue inventada por el norteamericano Robert S. Hamilton, quien en su libro Estado Presente de la Filosofía Social cuenta que en 1855 consultó a un amigo sobre la propiedad y necesidad de acuñar un nuevo término para expresar el conjunto de ideas implicadas en el estudio de la sociedad, sugiriendo socialítica, socialística y sociología; el amigo no encontró aceptable ninguna de las tres, y el autor resolvió bajo su propia responsabilidad adoptar Sociología. Poco más tarde, halla que este término había sido utilizado como título de una obra de George Fitzhugh Sociología Para el Sur (1854); asimismo, este término también se había incluido en la octava edición de la Enciclopedia Británica (1852) bajo el título de Comunismo. Según Hamilton, este hecho demostró «como diferentes cerebros, sin concertarse de antemano, pero movidos por las mismas necesidades, actuando ampliamente en la misma época del mundo, son llevados con frecuencia a los mismos descubrimientos o invenciones, sea de pensamiento o de palabras...... (2).

Pero ¿qué diferenciaba a la sociología de todas aquellas reflexiones anteriores? Antes del nacimiento de la sociología, los filósofos y los teólogos se limitaban a describir o imaginar cómo debía ser la sociedad, pero ninguno intentó analizarla tal como era realmente. Para conseguir este objetivo, debía aplicarse el método científico al estudio de la sociedad, que tantos resultados había dado en otras disciplinas (1).

\section{Origen de la sociología médica}

En la antigüedad, Hipócrates y Galeno, y después Paracelso, desarrollaron claras recomendaciones y criterios de observación que alertaban acerca del peso de los factores sociales sobre la salud de la población. Cuando Paracelso y luego Ramazzini sientan las bases de lo que posterior- mente será la medicina ocupacional, estaban indicando la estrecha relación entre organización económica y condiciones de salud (4).

El médico William Petty sostenía en el siglo XVII que la estructura demográfica, los factores sociales, económicos y culturales estaban en estrecha correspondencia histórica con la situación de salud. Esto significaba que, en la sociedad inglesa se percibía de manera clara y conflictiva la relación entre problemas sociales y los de la salud; bastaba revisar las novelas de Charles Dickens y la manera como se mostraba la explotación laboral de mujeres y niños en la temprana era industrial inglesa (5).

En el siglo Xix, la enfermedad se desarrollaba por las condiciones sociales y por factores del ambiente físico. Los estudios epidemiológicos desarrollados en el marco del movimiento de la medicina social, trataron precisamente el problema de las relaciones entre las enfermedades y los factores sociales. Es a propósito de estos trabajos que se ha habla por primera vez de Sociología Médica. Sin embargo, la participación del sociólogo había sido en ellos como auxiliar; al parecer jugó un papel más importante por su competencia en materia de investigación que por la introducción de una problemática original o de herramientas conceptuales nuevas $(6,7)$.

En 1848, Rudolf Virchow postula en Alemania la naturaleza del proceso salud-enfermedad como base conceptual para la elaboración de una política de salud para su época. Especialmente con su activismo político en los movimientos de reforma que culminan en las revoluciones de ese mismo año, Virchow inicia una serie de investigaciones influyentes referentes a los efectos de las condiciones sociales en la enfermedad y en la mortalidad. Además, presenta observaciones patológicas y datos estadísticos, y discute que la solución a estos problemas requiere un cambio social fundamental. Virchow define el nuevo campo de la medicina social como "ciencia social" $(4,8)$.

Años después, otros trabajos identificaron la importancia de los factores sociales, económicos y políticos relacionados al tema de salud. Tales es el caso de Federico Engles (1894) sobre las condiciones de la clase obrera en Inglaterra y del sociólogo francés Emile Durkheim (1897) con su obra El Suicido. Otros trabajos sobre la relación entre la medicina y la sociedad fueron el de la primera mujer licenciada en medicina en los Estados Unidos Elizabeth Blackwell «"Ensayos sobre sociología médica” en 1902, y el de James Warbasse "Sociología médica: una serie de observaciones que conciernen a la sociología de la salud y las relaciones de la medicina con la sociedad» en 1909 . Sin embargo, estas publicaciones eran fruto del trabajo de personas más preocupadas por la medicina que por la sociología $(6,9,10)$. 
Al parecer, quien utilizó por primera vez el término "Sociología Médica" fue el médico norteamericano Charles McIntire en 1893, en una conferencia en la Academia de Medicina en Milwauke (Estados Unidos) titulada The importance of the study of medical sociology. A pesar de su juventud, esta disciplina había sufrido un crecimiento intelectual impresionante. En diferentes puntos académicos de los Estados Unidos se comienza a abordar la investigación sobre los factores socio-culturales en la salud. Algunas obras que se escribieron en los inicios de esta disciplina son textos de consulta en la actualidad, entre ellos están el informe Flexner sobre Medical Education in the United States and Canada (1910); la historia de la medicina de Fielding Hudson Garrison (1929); Social Factors in Medical Progress de Bernard Stern (1927); Médico y el Paciente como Sistema Social Lawrence Henderson (1935); el análisis de la longevidad por Louis I. Dublin y Alfred J. Lotka (1936); el libro de Edgar Sydenstricker sobre Health and Environment (1933) y Robert E. Lee Faris y H. Warren Dunham sobre la esquizofrenia (1939). Pero el interés concreto por el estudio de los factores socio-culturales en la salud humana se desarrolla a partir de 1930 en varios lugares a la vez de los Estados Unidos: en Nueva York con los proyectos de sociología de la educación médica y en la Universidad de Yale con los estudios epidemiológicos y antropológicos aplicados a la comunidad de New Haven. Después de la década de los 50, las publicaciones sobre sociología médica se hacen cada vez más conocidas y de interés en el análisis de la salud. Así se tiene a Robert Strauss, The Nature and Status of Medical Sociology (1957); Henry E. Sigerist, Sigerist On the Sociology of Medicine (1960); David Mechanic, Medical Sociology (1968); Rodney M. Coe, Sociology of Medicine (1970); E. Gartly Jaco, Patients, Physicians, and Illness (1972); Howard H. Freeman, Sol Levine y Leo G. Reeder, Handbook of Medical Sociology (1979) (1,10-17).

La sociología médica en sí, surge en los Estados Unidos después de la segunda guerra mundial, cuando se consigue una importante financiación de dicho gobierno para la investigación socio médica, debido al interés por el estudio de la influencia de los factores socio-culturales en la etiología de la enfermedad mental como consecuencia del cambio social, estrés y sobre todo a la prevalencia (aparentemente muy alta) de desórdenes psiquiátricos diagnosticados durante la selección de la población masculina para el servicio militar. A su vez, muchos psiquiatras tuvieron que enfrentarse con variables de tipo social que habían condicionado la aparición de síntomas psiquiátricos durante la guerra, y especialmente durante los procesos de rehabilitación a la hora de tratar a sus nuevos pacientes. Lamentablemente, ésta sociología estuvo diri- gida más a problemas prácticos que al desarrollo teórico en sí. Sin embargo, esto se modificó con la publicación en 1951 de El Sistema Social de Talcott Parsons, en la cual se hace alusión a la práctica médica moderna y se acuñan los conceptos de rol del enfermo y el rol del médico. Para Parsons, un buen estado de salud es un prerrequisito funcional para que tanto un individuo como la sociedad puedan funcionar adecuadamente $(1,10,11,15,18)$.

Posteriormente Robert Strauss en 1957 (7, 9, 12, 1 5, 19) plantea que la sociología médica se divide en dos corrientes separadas, pero a la vez interrelacionadas por áreas, siendo estas:

a. Sociología en la medicina - también llamada sociología de la salud. El sociólogo colabora directamente con el médico y otros profesionales de la salud en el estudio de los factores sociales que son relevantes a un desorden de la salud en particular y estudia las causas y consecuencias de la salud y la enfermedad. El trabajo del sociólogo es la aplicación directa en el cuidado del paciente o en resolver problemas de la salud pública y analiza la etiología o causas de los desórdenes en salud, teniendo en cuenta que dichos desórdenes están relacionados a variables sociales como: edad, sexo, status socio-económico, raza, grupo étnico, educación y ocupación. Se caracteriza por la investigación y el análisis primeramente motivado por un problema médico más que por un problema sociológico. Asimismo recoge las perspectivas sociológicas, las teorías y los métodos y los aplica al estudio de la salud y la práctica médica. Los sociólogos en la medicina realizan trabajos aplicados en los cuales el conocimiento sociológico y los métodos de investigación se usan para proveer de información útil a los profesionales de la salud para mejorar la atención médica; a las organizaciones de la salud para ofrecer servicios más efectivos; y a los organismos de planificación y decisión para desarrollar programas y políticas tendientes a mejorar las condiciones de la salud en la sociedad.

b. Sociología de la medicina - se refiere a factores tales como organización, rol de las relaciones, normas, valores y creencias de la práctica médica como forma de comportamiento humano. El énfasis está dado en el proceso que ocurre en el escenario médico y como este contribuye al entendimiento de la sociología médica y en particular de la vida social en general. Se caracteriza por la investigación y el análisis del ambiente médico desde una perspectiva social.

Utilizan el conocimiento sociológico y los métodos de investigación para explicar, generalizar y predecir conductas sociales humanas en relación a situaciones de salud con el fin de desarrollar, testear y verificar teoría 
sociológica y contribuir al conocimiento general de la disciplina. Los sociólogos de la medicina comparten las mismas metas que otras áreas de la sociología ya que estudian fenómenos sociales como el poder, el conflicto, etc., en el ámbito de la salud y la enfermedad, desde el punto de vista de las preguntas sociológicas más que de los aspectos médicos.

Los primeros que se interesaron en el área de la sociología de la medicina fueron los sociólogos generales, quienes utilizaban los datos y las instituciones médicas para demostrar teorías sociológicas; generalmente sólo enseñaban a estudiantes de sociología.

Algunos autores mencionan que llama la atención el hecho de que a mediados de la década de los 50, los médicos más cercanos a los objetivos intelectuales de los sociólogos de la medicina solían ser paradójicamente los psiquiatras, aquellos que menos se identificaban con la sociología dura. Por otro lado, el desarrollo de la sociología de la medicina en Europa era incipiente y apenas se conocía salvo en el Reino Unido, Francia, Bélgica y Polonia, siendo uno de los programas europeos de sociología médica mejor desarrollados el de la Universidad Católica de Lovaina en Bélgica (20).

En 1960 la sociología de la medicina fue aceptada como una sección de la American Sociological Association (ASA) demostrando ser la sección más numerosa de todas. En 1966 se crea el Journal of Health and Social Behavior (JHSB) como una de las revistas oficiales de la As A $(6,21)$ En esa misma década, en un seminario organizado por la Organización Mundial de la Salud (oms) en Hannover (Alemania) en octubre de 1969, sobre el tema de Las ciencias sociales en la educación médica, se señala que: «Hasta muy recientemente los sociólogos, que habían estudiado sistemáticamente sistemas de conducta con la familia, la religión, la política, la economía ignoraban casi totalmente el sistema sanitario y médico..... Las ciencias sociales reflejaban la tendencia global de la sociedad a dejar a la medicina aparte, una actitud tradicional de las sociedades humanas que está asociada con el estatus carismático del médico» (22).

En la década de los 7os, la sociología médica estaba más desarrollada en los Estados Unidos que en cualquier otra parte del mundo. Los norteamericanos lideraban la investigación y las publicaciones sobre el tema. Asimismo, este campo se estaba haciendo cada vez más fuerte en Gran Bretaña y Alemania y estaba surgiendo en Israel, Japón, el resto de Europa y en América Latina. Hacia 1972 existían en los Estados Unidos 47 universidades que enseñaban sociología de la medicina a nivel de estudiantes graduados $(10,13,21)$.

En la década de los 80 en en los Estados Unidos, las ciencias sociales (primero la sociología, luego la antropología y por último la psicología) habían podido poco a poco captar un lugar en el campo de la salud. Estos estudios generalmente estaban dirigidos por médicos, especialistas en salud pública, medicina preventiva o medicina comunitaria. Sin embargo, posteriormente los profesionales de la salud aceptaron a la sociología porque se dieron cuenta que poseía una metodología que era gran utilidad, especialmente en el diseño de encuestas y en el análisis e interpretación de los datos.23 Asimismo, en 1986 la American Sociological Association (ASA) define la sociología médica como «una sub-disciplina que aplica las perspectivas, conceptos, teorías y metodologías de la sociología para estudiar fenómenos relacionados con la salud humana y la enfermedad» (24).

A comienzos de la década de 1990, Estados Unidos y Canadá contaban con 143 Escuelas de Medicina y con 1918 Departamentos de Sociología, cuyos profesores enseñaban en la mayoría de Escuelas de Medicina. En el caso del Reino Unido, en 32 de las 34 Escuelas de Medicina se enseñaba sociología médica. Al respecto, vale la pena resaltar que el tipo de sociología médica que enseñaban los sociólogos, estaba fuertemente condicionada por el departamento al que pertenecían, ya sea psiquiatría, medicina social, salud pública, epidemiología, medicina comunitaria, etc. $(25,26)$.

\section{Campos de la Sociología Médica}

No existe unanimidad entre los sociólogos y los índices de los libros varían constantemente. Es por ello, que algunos autores (12,27-29) mencionan que la disciplina puede dividirse en numerosos campos como:

A. Teoría - incluye tres tipos diferentes de aportes: 1- los libros de texto sobre sociología de la medicina; 2-las contribuciones de la historia de la medicina y de la salud pública y 3-algunas contribuciones teóricas globales. Dentro de este último grupo pueden incluirse las teorías sobre la salud y la medicina: la etnometodología, el interaccionismo simbólico, la teoría de roles, de etiquetas sociales, de desviación y de control social. También forman parte de esta sección la evolución de la disciplina y el desarrollo de la medicina desde la prehistoria hasta la medicina actual; incluyendo los temas de la innovación y el cambio social en la medicina.

B. Metodología - trata del análisis de las formas de influencia de los factores socio-culturales en la salud. Incluye los aportes de la epidemiología y hace referencia a los estudios de comunidades concretas. También se incluye los análisis respecto de la mortalidad y morbilidad, con sus problemas de medida, pruebas y escalas. Finalmente, se suman diversos modelos metodológicos como la investigación demográfica-epidemiológica, el análisis de datos secundarios, la encuesta y la experimentación. 
C. Enfermedad - incluye el estudio concreto de los factores culturales, psicológicos y sociales en la etiología de los desórdenes somáticos y psicosomáticos. Incluye también los diversos enfoques sobre las nociones y conceptos de salud y enfermedad, las teorías sobre la conducta desviada y el análisis del estrés.

D. Pacientes - reúne el análisis de pacientes individuales, grupos de pacientes y el tema del rol del enfermo, además de temas concretos como rehabilitación, casos terminales y etapas de la enfermedad. También se observa la educación de la salud, la higiene, la conducta del paciente y las reacciones de la familia ante la incidencia de la enfermedad. El componente actitudinal aquí es básico: antes, durantes y después de ser paciente.

E. Médicos - los temas clásicos son la educación médica, los tipos de médicos, su especialización, la organización de la profesión médica, la estructura de las asociaciones profesionales médicas, la relación médico-paciente. Obviamente se debe incluir aquí todo el personal de salud: enfermeras, comadronas, personal para-médico, curanderos, etc. Se debe agregar también el estudio concreto de la práctica médica, de la investigación médica y de otros profesionales de la salud.

F. Hospitales - se refiere a todo tipo de establecimientos de salud e incluso a consultas médicas. El análisis clásico incluye el desarrollo histórico de los hospitales, el complejo médico-industrial, la administración hospitalaria y los administradores, la estructura y la dinámica de los hospitales y las relaciones personales y de equipos. En general, incluye todo análisis sobre los entornos sociales en los que se realiza la práctica médica.

G. Comunidad - se estudia el tema de la educación médica de la población en general y las organizaciones de salud; es decir, todo lo que queda englobado en el ámbito general de la salud pública. Incluye relaciones con la estructura social y los factores culturales (religión, educación, pobreza, etc.). En general comprende todo lo referente al logro de la salud a nivel de la comunidad, junto con estudios epidemiológicos localizados especialmente.

H. Sociedad - el tema es más amplio y general. Incluye el estudio de los sistemas de salud nacionales en relación con la estructura y cambio sociales, el tema de planificación en salud (objetivos, alternativas, prioridades, toma de decisiones, implementación y evaluación), el costo y financiación de la salud (seguro de salud), la política de salud nacional, la socialización de la medicina, las relaciones con la salud internacional, los problemas del personal médico y las relaciones entre desarrollo económico y salud. Un modelo de sistema de salud es el objetivo principal que se busca en este punto.
De otro lado, para Schuffer, (7) las preguntas típicas de un sociólogo que investiga en el área de la medicina son: ¿qué factores sociales, tales como clase social, estilos de vida, afectan la salud y la enfermedad?, ¿cuáles son las funciones sociales de las instituciones y organizaciones de salud?, ¿cuál es la relación entre los sistemas de atención de la salud y otros sistemas sociales?, ¿qué estilos de conducta social caracterizan al personal y a los usuarios del área de salud?, entre otros. Según Schufer,(4) la sociología médica tiene su objetivo propio: el estudio de las definiciones sociales de la enfermedad; de las prácticas que se relacionan con ella y de las instituciones que la toman a su cargo; y del estatus social y de los comportamientos del enfermo.

Asimismo, otros autores, $(15-17,30)$ mencionan diversas aplicaciones de la sociología en la medicina, las cuales son:

- distribución, prevención y diagnóstico de las enfermedades en la población

- actitudes y creencias de los pacientes, médicos y profesionales de la salud sobre la salud y la enfermedad

- qué hacen las personas para mantener, mejorar o recuperar su salud

- como se organiza, funciona y financia la profesión medica

- acceso a los servicios médicos

- respuesta y adaptación a la enfermedad

- rol de la medicina como institución social y su relación con otras instituciones

- rol de las variables sociales en la etiología (el estudio de las causas de las enfermedades)

- tratamiento y rehabilitación del individuo donde tiene gran importancia la familia

- relación médico-paciente (confianza)

- uso de técnicas de investigación de las ciencias sociales dentro de la medicina

- información y mensajes en los medios masivos de comunicación

- conocimiento del personal médico sobre la evolución y desarrollo de la sociedad con relación a la salud

- historia social de la enfermedad

- estadísticas relacionadas con aspectos socioeconómicos y demográficos que se utilizan en medicina y salud pública Para concluir, e enfoque de la sociología en la medicina fue aceptado cuando planificadores y gobernantes se dieron cuenta de que, a pesar de los grandes descubrimientos médicos del siglo pasado, la reducción de la mortalidad se debía fundamentalmente al desarrollo de la salud pública mediante la educación de la población, urbanización y desarrollo económico. En tal sentido, no es exagerado asegurar que durante el último siglo, las ciencias sociales han contribuido a salvar más vidas que las ciencias médicas (31). 
En el futuro, la institucionalización de la sociología médica dependerá de las condiciones socio-políticas y de la estructura universitaria. Si existen facultades de sociología, éstas se institucionalizarán con independencia de las facultades de medicina. Los recursos para la investigación provendrán del consumidor de servicios sanitarios, la industria farmacéutica, la seguridad social y fundaciones privadas (23).

Finalmente, a largo plazo la prevención de enfermedades a través de cambios de conducta y estilos de vida, tanto a nivel individuo como a nivel de la comunidad, deberá basarse en la utilización de las ciencias sociales, especialmente la sociología y la antropología. Para ello, la medicina deberá trabajar de manera complementaria con las ciencias sociales, ya que éstas pueden ayudar a explicar determinados aspectos relacionados a la salud que no forman parte del estudio de la medicina (23)

\section{REFERENCIAS}

1. Macionis JJ, Ken Plummmer K. La perspectiva sociológica. En: Sociología. Madrid: Pretince Hall; 1999.

2. Hernández-Urbina A. Origen de la sociología. En: Compendio de Sociología Peruana. Lima: Ediciones Ruiz, Segunda Edición; 1966.

3. Comte A. A Dictionary of Sociology. 3er. Edition, John Scott \& Gordon Marshall (eds): Oxford University Press; 2005.

4. BritoQuintana P. La incorporación de las ciencias sociales en la enseñanza de las ciencias de la salud: una historia conflictiva. En: Aplicaciones de las Ciencias Sociales en Programas de Salud. John Sparkman Center for International Public Health Education, The University of Alabama at Birmingham, Agustín Montoya de la Cadena, David W. Coombs y Oscar Henao Cabal (eds.), Sparkman Center Series in International Health, Number One; 1988.

5. Echeverrí-Hernández JV. Relaciones entre las ciencias sociales y el campo de la salud. Revista de Psicología 2010; 2(4):47-55.

6. Duarte-Nunes E. La importancia del estudio de la sociología médica: un texto del siglo XIX. Revista de la Sociedad Española de Historia de las Ciencias y de las Técnicas 1994; 17(33):313-324.

7. Schuffer-ML. Aportes de la sociología a la medicina: una perspectiva histórica. Medicina y Sociedad 1993; 1(16):13-18.

8. Waitzkin H. The social origins of illness. In: The Second Sickness: Contradictions of Capitalist Health Care. 2nd ed: Lanham, Rowman \& Littlefield; 2000.

9. Briceño-León R. Las ciencias sociales y la salud: un diverso y mutante campo teórico. Ciênc. Saúde Coletiva 2003; 8(1):33-45.
10. Cockerman WC. Medical sociology at the millennium. In: Medical Sociology. Major themes in health and social welfare. New York: Routledge; 2005.

11. El desarrollo de la sociología en la medicina. [Internet]. Disponible en: https://goo.gl/9DPdnV. . Consultado agosto del 2014.

12. De Miguel J. Fundamentos de la sociología de la medicina. En: Sociología de la Medicina: Una Introducción Crítica, Ed. Vicens-Vives, 1ra. Edición, Barxeloina: Vincens Universidad; 1978.

13. Duarte-Nunes E. As ciências sociais em saúde: reflexões sobre as origens e a construção de um campo de conhecimento. Cadernos de Saúde Pública 1992; 1(1):59-84.

14. Faris R, Dunham HW. Mental Disorders in Urban Areas. The University Chicago Press;1939.

15. Fox R. Reflexions and opportunities in the sociology of medicine. J. of HIth. Soc. Behav. 1985; 26:6-14.

16. García JC. Ciencias sociales y salud. República Dominicana: Colección Sespas; 1981.

17. González I. Sociología médica o sociología de la salud. [Internet]. Disponible en https://goo.gl/ihBkMW. Consultado agosto de 2014.

18. Suchman EA. Sociology and the Field of Public Health. New York: Rusell Sage Foundation; 1963.

19. Cockerham WC. Medical Sociology. Preantice Hall Series in Sociology: Englewood Cliffs; 1978.

20. Goss MEW, Reader GG. Collaboration between sociologist and physician. Social Problems 1956; 4:82-89.

21. Hollingshead AB. Medical sociology: a brief review. Quarterly Health and Society 1973; 51(4); 531-542.

22. World Health Organization. The Social Sciences in Medical Education. Copenhague; 1970.

23. Coombs DW. Introducción. En: Aplicaciones de las Ciencias Sociales en Programas de Salud, John Sparkman Center for International Public Health Education, The University of Alabama at Birmingham, Agustín Montoya de la Cadena, David W. Coombs y Oscar Henao Cabal (eds.), Sparkman Center Series in International Health, Number One; 1988.

24. Pacheco J, González I. Sociología de la salud. Primera Clase. [Internet]. Disponible en: https://goo.gl/HgjREc. Consultado agosto 2014.

25. Espinel-Vallejo M. La sociología de la salud de espacio europeo de educación superior en España: estudio preliminar. [Internet]. Disponible en: https://goo.gl/HZzpok. Consultado agosto de 2014.

26. Hunt G, Sobal, J. Teaching Medical Sociology in Medical Schools. Teaching Sociology 1990; 18(3):319-328.

27. Chaiklin $\mathrm{H}$. The state of the art in medical sociology. The Journal of Nervous and Mental Disease 2011; 199(8):585-491.

28. Coe RM. Sociology of Medicine. New York: McGraw-Hill; 1970.

29. Mechanic D. Medical Sociology. New York: Free Press; 1968.

30. Saxon G. Cancer, culture and social structure, En: Patients, Physicians and IIIness. F. Gartly Jaco (comp), New York: Free Press; 1972.

31. Eisenberg L, Kleinman A, eds. The Relevance of Social Science for Medicine. Dordrecht:D. Reidel Publishing Co.; 1981. 